\title{
Apnoea of prematurity - discontinuation of methylxanthines in a resource-limited setting
}

\author{
L J Tooke, ${ }^{1}$ MB ChB, FCPaeds, MMed (Paeds), Cert Neonatol (SA); K Browde, ${ }^{2}$ MB ChB; \\ M C Harrison, ${ }^{1} \mathrm{MB} \mathrm{ChB}, \mathrm{MRCP}, \mathrm{FRCPCH}$ \\ ${ }^{1}$ Neonatal Department, Groote Schuur Hospital, and Department of Paediatrics, University of Cape Town, South Africa \\ ${ }^{2}$ Department of Paediatrics, Red Cross War Memorial Children's Hospital and University of Cape Town, South Africa
}

Corresponding author: L Tooke (lloyd.tooke@uct.ac.za)

Background. Methylxanthines such as caffeine have been proven to reduce apnoea of prematurity and are often discontinued at 35 weeks corrected gestational age (GA).

Objective. To ascertain whether a caffeine protocol based on international guidelines is applicable in our setting, where GA is often uncertain Methods. A prospective folder review was undertaken of all premature infants discharged home over a 2-month period.

Results. Fifty-five babies were included. All babies born at less than 35 weeks' GA were correctly started on caffeine as per protocol. GA was assigned in $85.5 \%$ of cases by Ballard scoring and in $14.5 \%$ from antenatal ultrasound findings. Caffeine was discontinued before 35 weeks in $54.5 \%$.

Discussion. The main reason for discontinuing caffeine early was the baby's ability to feed satisfactorily, a demonstration of physiological maturity. As feeding behaviours mature significantly between 33 and 36 weeks, the ability to feed may be a good indication that caffeine therapy can be stopped.

S Afr J CH 2013;7(4):146-147. DOI:10.7196/SAJCH.630

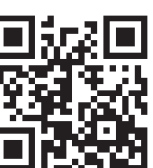

Apnoea of prematurity (AOP) is a complete cessation of breathing that occurs as a result of immaturity of respiratory control in preterm infants and is considered a physiological rather than pathological process. It is most commonly defined as a pause in breathing that lasts more than 15 seconds or one that is associated with desaturation and bradycardia. ${ }^{[1]}$

AOP remains among the most commonly diagnosed conditions in the newborn intensive care unit. ${ }^{[2,3]}$ The incidence of AOP is inversely correlated with gestational age (GA). In infants who are born at $<29$ weeks' gestation, AOP is almost universal, while at 35 weeks only $7 \%$ of infants will have AOP. ${ }^{[1]}$

Treatment modalities for AOP include continuous positive-pressure ventilation and methylxanthines. The two methylxanthines most commonly used are caffeine and theophylline (and aminophylline, the intravenous form of theophylline) ${ }^{[4]}$ Cochrane meta-analysis has shown that there is a significant reduction in apnoeic episodes and subsequent mechanical ventilation in infants treated with methylxanthines. ${ }^{[5]}$

Although theophylline is effective, caffeine has been shown to be the faster-acting and safer of the two drugs, with a wider therapeutic range and fewer side-effects. It also has a longer half-life (up to 100 hours) and so requires only once-daily dosing. ${ }^{[6,7]}$

Schmidt et al. ${ }^{[8,9]}$ showed a significant reduction in the rate of bronchopulmonary dysplasia and an improvement in neurodevelopmental outcome at 18 - 21 months in infants treated with caffeine ${ }^{[8,9]}$ However, this neurodevelopmental advantage could not be demonstrated at 5 years of age. ${ }^{[10]}$

As a result of its proven benefits and cost-effectiveness, caffeine is now one of the most widely used drugs in neonatology. ${ }^{[11]}$ Despite being on the World Health Organization Essential Drugs List for children, it is unavailable in many low-resourced settings owing to cost and manufacturing problems. ${ }^{[4]}$

Although caffeine prophylaxis as opposed to treatment has not been definitively proven to be effective ${ }^{[5]}$ many units give prophylaxis to infants who have the highest risk of apnoea. ${ }^{[12]}$ Caffeine is generally stopped at 33 - 35 weeks' postmenstrual age. ${ }^{[8,12]}$

\section{Objective}

To ascertain whether the caffeine protocol based on internationa guidelines is applicable in our setting.

\section{Methods}

Groote Schuur Hospital is a tertiary referral hospital in Cape Town, South Africa. The neonatal unit treats over 500 very-low-birth-weight infants $(<1500 \mathrm{~g})$ per year. Owing to socio-economic circumstances, very few of the mothers have had early ultrasound scans and the GA of the babies is usually estimated using the modified Ballard Score. ${ }^{[14]}$

The Groote Schuur nursery caffeine protocol was developed using international guidelines. Since our high patient-to-nurse ratio results in some apnoeas not being detected, it was decided to start all infants of $<35$ weeks' GA at birth on caffeine prophylactically. The protocol states that the caffeine dose should be adjusted for weight and discontinued when the corrected GA is 35 weeks, provided there have been no apnoeic episodes in the previous week. Caffeine base is the solution used in our nursery, and after a loading dose of $10 \mathrm{mg} /$ $\mathrm{kg}$, a daily dose of $5 \mathrm{mg} / \mathrm{kg}$ is used.

In a prospective folder review done over the months of December 2011 and January 2012, we reviewed the folders of all premature babies discharged from the nursery. Information was obtained from the infant records and medication charts and recorded on a structured data pro forma. Data collected included GA at birth and how this was ascertained, caffeine prescriptions, reasons for caffeine discontinuation, and apnoeic events. Permission for the study was obtained from the Faculty of Health Sciences Research Ethics Committee.

\section{Results}

Fifty-five babies fulfilled our inclusion criteria. All babies born at $<35$ weeks' gestation were started on caffeine as per protocol. Two patients who were scored at 36 weeks were also started on caffeine. One of these was given caffeine as treatment for apnoea, while the other was given caffeine in error. GA was assigned in 47 (85.5\%) of the babies using the modified Ballard Score and in 8 (14.5\%) from ultrasound findings. 
We found that nearly $60 \%$ of the infants receiving caffeine in our unit did not have their doses increased as they gained weight. In only 18 out of the 55 patients (32.7\%) was caffeine discontinued at 35 weeks' corrected GA as per protocol. In the majority of the babies $(54.5 \%)$, caffeine was discontinued earlier than the guidelines recommended (Fig. 1).

The main reason (60.0\%) given for stopping caffeine early was that the babies were cup feeding or breastfeeding and therefore getting ready to go home or into kangaroo mother care (Fig. 2). Continuing to give caffeine after 35 weeks (12.7\% of babies) was either due to concerns about ongoing apnoea or failure to stop the medication according to protocol.

There were no recorded apnoea events after caffeine was stopped.

\section{Discussion}

The study revealed that the caffeine dose was not appropriately adjusted as the weight increased in the majority of babies (60\%). This finding highlights the need for vigilance in reviewing dosages of not only caffeine but all long-term medications where the dosage is based on weight.

In $55 \%$ of the babies, caffeine was stopped at a lower GA than recommended by the protocol. This was due to their imminent discharge, as they showed signs of physiological maturity such as the ability to feed satisfactorily. As we do not discharge babies on caffeine, it is usually discontinued at least 5 days before discharge. Studies have shown that feeding behaviour in premature infants matures significantly between 33 and 36 weeks after conception, and swallowing infrequently interrupts respiration during feeding after 35 weeks after conception. Babies who are unwell or have respiratory or neurological complications may have a delay in developing the ability to feed. ${ }^{[13]}$

Ballard scoring can misjudge GA by up to 2 weeks even when the scorer is experienced. ${ }^{[14]}$ Less experienced doctors have even greater margins of error. Also, many of our babies are growth restricted, ${ }^{[15]}$ which often confounds GA scoring as smaller babies may be scored as younger. ${ }^{[16]}$

Reassuringly, no babies who had their caffeine stopped because they were able to feed subsequently developed apnoea.

\section{Conclusion}

In our setting, GA is often difficult to estimate and the potential margin of error is wide. Ability to feed rather than the estimated GA may therefore be the better indicator to stop administration of caffeine or other methylxanthines. Stopping methylxanthines based on feeding could result in less unnecessary therapy and even shorten hospital stay.

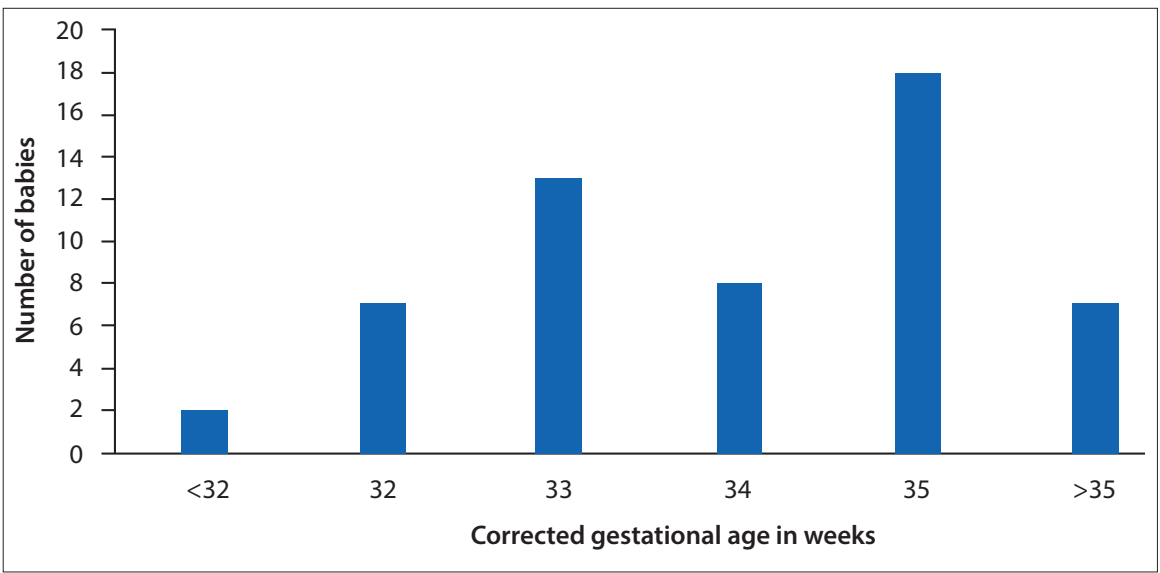

Fig. 1. Corrected gestational ages when caffeine was discontinued.

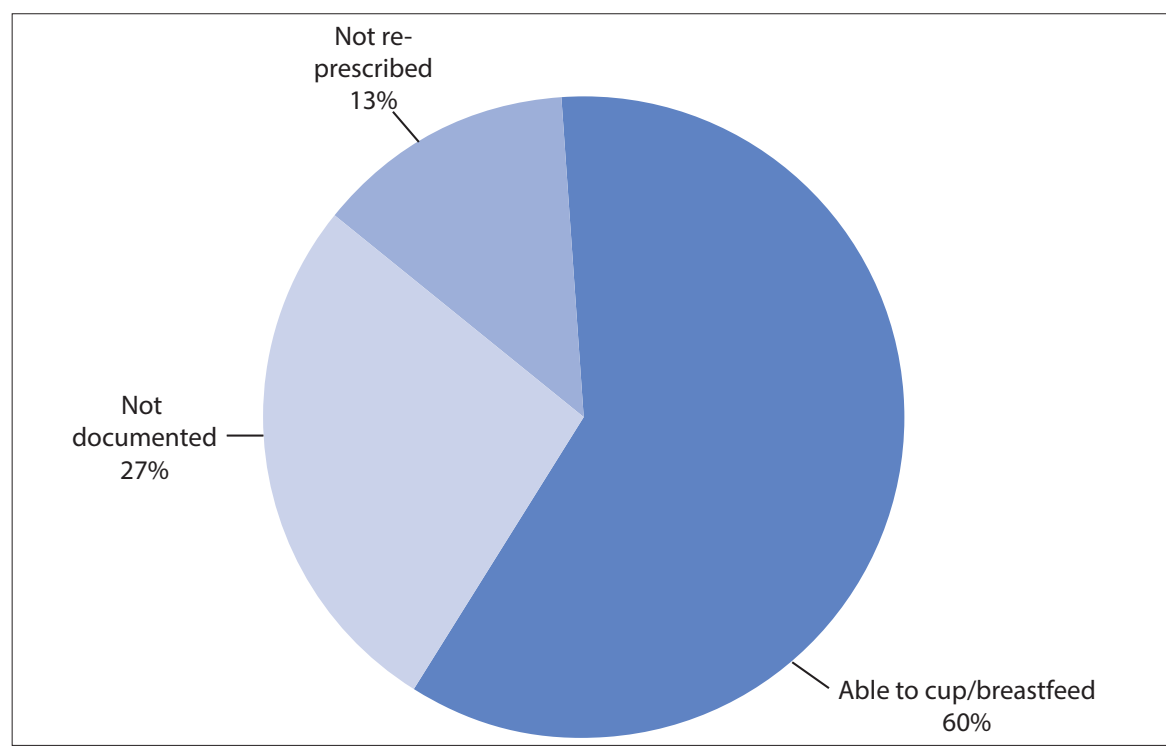

Fig. 2. Reasons for discontinuing caffeine before 35 weeks.

\section{References}

1. Zhao J, Gonzalez F, Mu D. Apnea of prematurity: From cause to treatment. Eur J Pediatr 2011;170(9):1097 1105. [http://dx.doi.org/10.1007/s00431-011-1409-6]

2. Eichenwald EC, Abimbola A, Stark AR. Apnea frequently persists beyond term gestation in infants delivered at 24 to 28 weeks. Pediatrics 1997;100(3);354359. [http://dx.doi.org/10.1542/peds.100.3.354]

3. Orozco-Gregorio H, Mota-Rojas D, Villanueva D, et al. Caffeine therapy for apnoea of prematurity: Pharmacological treatment. African Journal of Pharmacy and Pharmacology 2011;5(4):564-571.

4. Mueni E, Opiyo N, English M. Caffeine for the management of apnea in preterm infants. Int Health 2009;1(2):190-195. [http://dx.doi. org/10.1016/j.inhe.2009.09.005]

5. Henderson-Smart DJ, De Paoli AG. Methylxanthine treatment for apnoea in preterm infants. Cochrane Database Syst Rev 2010;12. Art. No. CD000432. [http:// dx.doi.org/10.1002/14651858.CD000140.pub2]

6. Laubscher B, Greenough A, Dimitriou G Comparative effects of theophylline and caffeine on respiratory function of prematurely born infants. Early Hum Dev 1998;50(2):185-192.

7. Scanlon JE, Chin KC, Morgan ME, Durbin GM, Hale KA, Brown SS. Caffeine or theophylline for neonatal apnoea? Arch Dis Child 1992;67(4):425-428. [http:// dx.doi.org/10.1136/adc.67.4 Spec No.425]

8. Schmidt B, Roberts RS, Davis P, et al. Caffeine therapy for apnea of prematurity. N Engl J Med 2006;354(20):2112-2121. [http://dx.doi.org/10.1056/ NEJMoa054065]
9. Schmidt B, Roberts RS, Davis P, et al. Long-term effects of caffeine therapy for apnea of prematurity. N Engl J Med 2007;357(19):1893-1902. [http:// dx.doi.org/10.1056/NEJMoa073679]

10. Schmidt B, Anderson P, Doyle L, et al. Survival without disability to age 5 years after neonatal caffeine therapy for apnea of prematurity. JAMA 2012;307(3):275-282 [http://dx.doi.org/10.1001/jama.2011.2024]

11. Dukhovny D, Lorch SA, Schmidt B, et al. Economic evaluation of caffeine for apnea of prematurity. Pediatrics 2011;127(1):e146-e155. [http://dx.doi org/10.1542/peds.2010-1014]

12. Martin R. Management of apnea of prematurity In: Garcia-Prats JA, ed. UpToDate. Waltham, Mass: UpToDate, 2012.

13. Mizuno K, Ueda A. The maturation and coordination of sucking, swallowing, and respiration in preterm infants. J Pediatr 2003;142(1):36-40. [http://dx.doi. org/10.1067/mpd.2003.mpd0312]

14. Ballard JL, Khoury JC, Wedig K, Wang L, Eilers-Walsman BL, Lipp R. New Ballard Score, expanded to include extremely premature infants. J Pediatr 1991;119(3):417-423. [http://dx.doi org/10.1016/S0022-3476(05)82056-6]

15. Lango MO, Horn AR, Harrison MC. Growth velocity of extremely low birth weight preterms at a tertiary neonatal unit in South Africa. J Trop Pediatr 2013;59(2):79-83. [http://dx.doi.org/10.1093/tropej/fms049]

16. Woods DL, Malan AF. Assessment of gestational age in twins. Arch Dis Child Health 1977;52(9):735737. [http://dx.doi.org/10.1136/adc.52.9.735] 